\title{
Successful Use of the Heart Ware HVAD as Bridge to Transplantation in an 8 Year Old Boy with a Previous History of Berlin Heart EXCOR Support
}

Raj Sahulee* and Hari Rajagopal

Department of Pediatrics, Division of Pediatric Cardiology, Icahn School of Medicine at Mount Sinai, New York, USA

\begin{abstract}
Continuous-flow ventricular assist devices are being implanted with increased frequency in the United States in children with end-stage heart failure. We report the first reported use of the HeartWare HVAD in an 8 year old boy who had a history of biventricular support with Berlin Heart EXCOR devices 4 years previously. He was supported on the HeartWare for 198 days prior to receiving a heart transplantation. The implantable HeartWare HVAD can be used to provide circulatory support for those who have had previous paracorporeal mechanical circulatory support.
\end{abstract}

Keywords: HeartWare HVAD; Mechanical circulatory support; Ventricular assist device; Pediatric; Children; Heart failure; Transplantation

\section{Introduction}

Dilated cardiomyopathy (DCM) is the final stage of various genetic, metabolic, and acquired conditions that affect the cardiac myocyte. For many children with DCM, heart transplantation (HT) is the most successful and long lasting therapy. As a bridge to HT, mechancal circulatory support (MCS), and specifically ventricular assist device (VAD) therapy is being used with increased frequency in adults and children. Ventricular assist device therapy potentially restores end-organ function, facilitates rehabilitation, and improves patient candidacy for HT. According to the PEDIMACS registry, 216 VADs were implanted in children in the United States from September 2012 to December 2014 [1]. Since the first reports in 2011, the continuous flow HeartWare HVAD (Heartware Inc, Framingham, MA) is being implanted in children with end stage heart failure with increasing frequency $[2,3]$. Unlike most continuous flow VADs that are only suitable for large adolescents and adults, the HeartWare HVAD is small enough to be implanted intrapericardially (Figure 1). The experience with the HeartWare HVAD in children still remains limited, and only a few reports describe the device's use in patients with previous MCS [4]. We report the first successful use of a HeartWare HVAD as a bridge to transplantation in a child who had previously been supported with biventricular Berlin Heart EXCOR (Berlin Heart $\mathrm{GmbH}$, Berlin, Germany) devices.

\section{Case Report}

A 4 year old Hispanic male with DCM and end stage heart failure secondary to fulminant myocarditis underwent successful implantation of biventricular Berlin Heart EXCOR devices after a 14 day stabilization period on extra-corporeal membrane oxygenation (ECMO). The patient was maintained on biventricular support with eventual significant recovery of biventricular function and explantation of the devices on post-operative day (POD) 15. Over the following 4 years, the patient had evidence of gradually deteriorating left ventricular $(\mathrm{LV})$ function and several episodes of supraventricular tachycardia (SVT). In May of 2014, at the age of 8 (23 Kg, BSA 0.86 $\mathrm{m}^{2}$ ), he presented with worsening of LV function with increased symptoms of end-stage heart failure including anorexia, abdominal pain and vomiting, and repeated episodes of symptomatic SVT. He was hospitalized and started on intravenous milrinone therapy without significant symptomatic improvement after 12 days. Echocardiography and cardiac catheterization confirmed severely depressed LV function (ejection fraction 26\%). Figure 2 with elevated mean filling pressures (RA $8 \mathrm{mmHg}$, LA $18 \mathrm{mmHg}$, PA $32 \mathrm{mmHg}$, PVR 4.4 woods units $/ \mathrm{m}^{2}$ ). With informed consent from the family, the patient was taken to the operating room and a HeartWare HVAD was implanted.

The device was implanted via median sternotomy using cardiopulmonary bypass via bicaval cannulation at normothermia. The site of the previous Berlin Heart cannulation of the LV was identified and avoided. The sewing ring for the HeartWare device was attached with running sutures and a strip of Teflon felt. The HeartWare's inflow cannula was then inserted through the sewing ring, aligned with the mitral valve and the pump was secured to the sewing ring (Figure 3). Due to previous operation, there was an old graft stump at the ascending aorta. A cardioplegic catheter was placed proximally from this and the

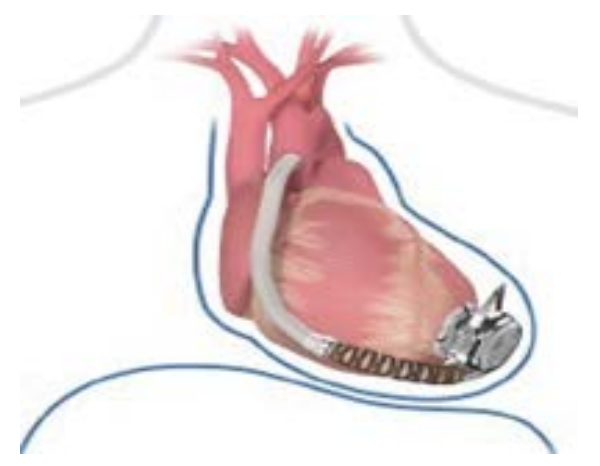

Figure 1: Schematic drawing of the HeartWare device after intrapericardial implantation.

*Corresponding author: Raj Sahulee, 1 Gustave L, Levy Place, Division of Pediatric Cardiology, New York, USA, Tel: 917-597-9250; E-mail: Raj. sahulee@mssm.edu

Received February 04, 2016; Accepted March 04, 2016; Published March 10 2016

Citation: Sahulee R, Rajagopal H (2016) Successful Use of the Heart Ware HVAD as Bridge to Transplantation in an 8 Year Old Boy with a Previous History of Berlin Heart EXCOR Support. J Cardiovasc Dis Diagn 4: 238. doi:10.4172/23299517.1000238

Copyright: (c) 2016 Sahulee R, et al. This is an open-access article distributed under the terms of the Creative Commons Attribution License, which permits unrestricted use, distribution, and reproduction in any medium, provided the original author and source are credited. 


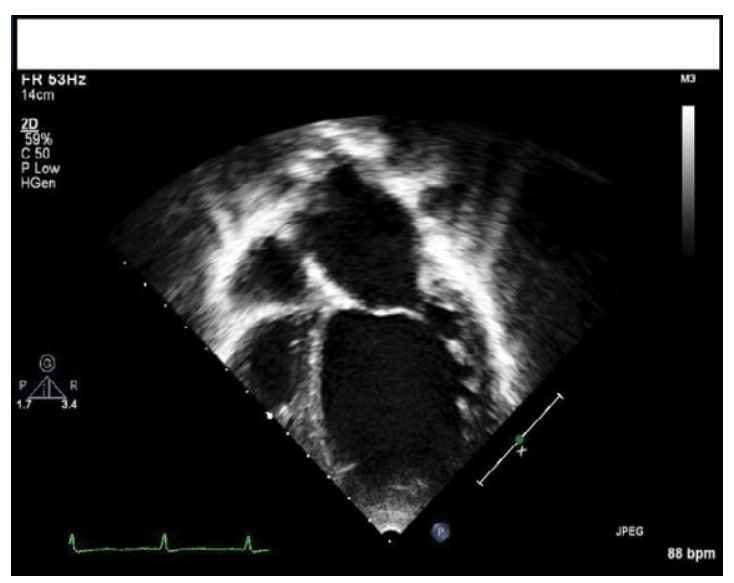

Figure 2: Dilated and dysfunctional left ventricle.

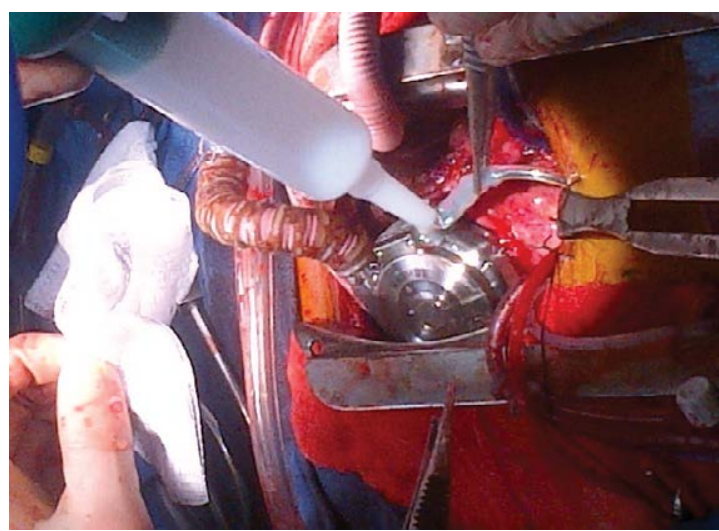

Figure 3: Heart Ware device after surgical implantation in the sewing ring

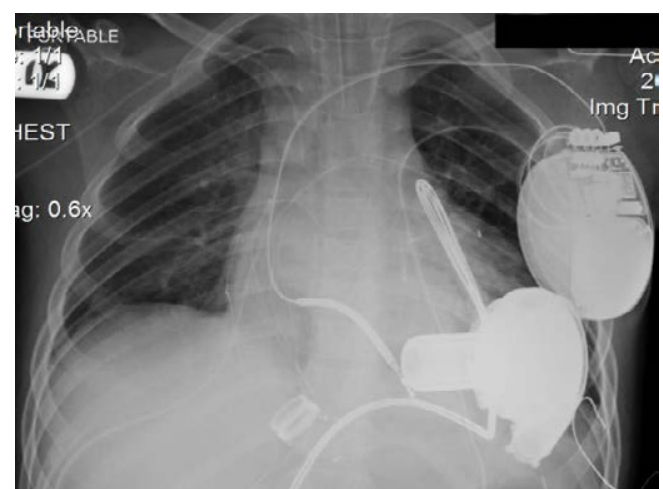

Figure 4: Radiographic appearance of the HeartWare device after implantation.

graft was anastomosed to the ascending aorta end-to-side fashion. Once this was accomplished, the device was activated and the patient was taken off bypass. After adjustment of the revolutions per minute (RPM) to 2600 on the device and titration of the inotropic support, adequate flow through the device of 3-3.5 L/min (cardiac index 3.5-4 L/ $\mathrm{min} / \mathrm{m}^{2}$ ) was achieved with an energy of 2.3 watts. The chest was closed briefly, but the right ventricle (RV) was noted to be dysfunctional on transesophageal echocardiogram. Therefore, the decision was made to leave the chest open. The mediastinum was covered with a piece of latex membrane and the patient was transferred back to cardiac intensive are unit in stable condition (Figure 4). In all, the total bypass time was 126 minutes for the procedure.

The postoperative course was complicated by significant bleeding and external compression of the right atrium and RV free wall requiring re-exploration on 2 separate occasions. However his sternum was finally closed on POD 10. During his post-operative course, several chest tubes were placed and remained present for several weeks for prolonged drainage. Additional complications included prolonged respiratory failure requiring tracheostomy, and poor oral nutrition requiring nasogastric nutritional supplementation. Cardiac output from the HVAD however remained well preserved at $2600 \mathrm{rpms}$ and cardiac output ranged from 3-4 L/min. Anticoagulation was maintained initially with heparin, and then transitioned to aspirin, coumadin and dipyramidole. The target international normalized ratio of 2-3 was achieved and there were no consistent evidence of either hemolyis or thrombosis from the device. Over several weeks the patient demonstrated recovery of all end-organ function including eventual tracheostomy decannulation. The patient underwent rehabilitation therapy and was able to ambulate without assistance. On POD 198, a suitable donor heart became available and the patient underwent orthotopic heart transplantation.

The patient's post-transplant course was uneventful. He underwent cardiac catheterization with biopsy which showed a good anatomic result, with normal filling pressures, normal LV function and no evidence of cellular or antibody mediated rejection. He was eventually discharged home with in-home rehabilitation on POD 17 after his transplant.

\section{Comments}

To date, this case represents the first successful use of Hearware HVAD as a bridge to transplantation in a patient with a history of previous implantation with biventricular Berlin Heart EXCOR devices. While medical therapy is sufficient for some patients with heart failure, there is a growing pediatric population who require $\mathrm{HT}$ who have been bridged to transplantation with various forms of MCS. Accordingly, the reported use of VAD therapy has increased dramatically in the pediatric population in the past 10 years [1]. Although current VADs allow for successful bridge to transplantation for congenital and acquired heart disease, there is limited experience in children implanted with the Heartware HVAD who were supported previously with other forms of MCS. Crews et al. reported successful HeartWare implantation after ECMO cannulation in a child [4], but there are no reports of the HeartWare HVAD being implanted in the scarred myocardium at the ventricular apex from a previous Berlin Heart EXCOR cannulation site. Because of the limited expericence with HeartWare HVAD implantation in young children with previous MCS, minor technical modificationswere used during implantation, but were not overly challenging. Prolonged bleeding and chest tube output was the only complication possibly related to previous apical cannulation. However the previous site of ventricular cannulation did not pose an obvious obstacle to successful device implantation and function.

The HeartWare device was utilized in our 8 year old patient with only a BSA of $0.86 \mathrm{~m}^{2}$. Currently the HeartWare HVAD is only recommended for patients with BSA of $1.5 \mathrm{~m}^{2}$ and greater (HeartWare, Inc), however it has been reportedly been successfully implanted in patients as small as a BSA of $0.70 \mathrm{~m}^{2}$ by Miera et al. [2]. Furthermore, 
at the time of this report, the longest reported case of HeartWare device support to successful transplantation in children in the US was 148 days [5]. Our patient was supported for 198 days without any significant thrombotic or hemorrhagic neurologic injury. Continuous flow devices like the HeartWare HVAD are being used in children with increasingly smaller BSA and to date this report is one of few that have been successful in bridging such a small child to transplantation after $>6$ months of VAD therapy.

Providing ventricular support with a continuous flow device provided the opportunity to more safely deliver increased cardiac output, with less risks of hemorragic or thrombotic complications when compared traditional pulsitile devices. Despite initial difficulties with bleeding after device implantation and prolonged respiratory failure requiring tracheostomy, the device provided excellent circulatory support. The HeartWare HVAD allowed us to greatly improve our patient's end organ function and symptoms, leading to tracheostomy decannulation, ambulation, rehabilitation and weight gain that made him a more ideal HT candidate.

Although the number of VADs used in older children and adolescents in heart failure has increased, the MCS options for infants remains limited, and currently the HeartWare HVAD is not applicable for that patient population. However, the Food and Drug Administration is still in the process of developing alternative devices in young patients in need of MCS. With the achievements in MCS in young children over the past 5-10 years, we are optomistic that novel devices will be developed and new applications for current devices will be utilized as potentially life-saving therapy for this unique and challenging population.

\section{References}

1. Kirklin JK, Naftel DC, Pagani FD, Kormos RL, Stevenson LW, et al. (2015) Seventh INTERMACS annual report: 15,000 patients and counting. J Heart Lung Transplant 34: 1495-1504.

2. Miera O, Potapov EV, Redlin M, Stepanenko A, Berger F, et al. (2011) First experiences with the HeartWare ventricular assist system in children. Ann Thorac Surg 91: 1256-1260.

3. D'Alessandro D, Forest SJ, Lamour J, Hsu D, Weinstein S, et al. (2012) First reported use of the heartware HVAD in the US as bridge to transplant in an adolescent. Pediatr Transplant 16: e356-359.

4. Crews KA, Kaiser SL, Walczak RJ, Jaquiss RD, Lodge AJ (2013) Bridge to transplant with extracorporeal membrane oxygenation followed by HeartWare ventricular assist device in a child. Ann Thorac Surg 95: 1780-1782.

5. Niebler RA, Ghanayem NS, Shah TK, Bobke ADLR, Zangwill S, et al. (2014) Use of a HeartWare ventricular assist device in a patient with failed Fontan circulation. Ann Thorac Surg 97: e115-116. 